\title{
Predictive value of early continuous amplitude integrated EEG recordings on outcome after severe birth asphyxia in full term infants
}

\author{
Lena Hellström-Westas, Ingmar Rosén, Nils W Svenningsen
}

\begin{abstract}
The background pattern in single channel amplitude integrated EEG recordings (aEEG) was recorded in 47 infants within the first six hours after birth to see if this could predict outcome after birth asphyxia.

The aEEG background pattern during the first six hours of life was continuous and of normal voltage in 26 infants. All these infants survived; 25 were healthy, one had delayed psychomotor development. A continuous but extremely low voltage pattern was present in two infants, both of whom survived with severe handicap. Five infants had flat (mainly isoelectric) tracings during the first six hours of life; four died in the neonatal period, and one survived with severe neurological handicap. Burst-suppression pattern was identified in 14 infants, of whom five died, six survived with severe handicap, and three were healthy at follow up. The type of background pattern recorded within the first six postnatal hours in the aEEG tracings predicted outcome correctly in 43 of $47(91 \cdot 5 \%)$ infants.
\end{abstract}

Use of aEEG monitoring can predict outcome, with a high degree of accuracy, after birth asphyxia, within the first six hours after birth. The predictive value of a suppression-burst pattern was, however, somewhat lower than the other background patterns. The aEEG seems to be a feasible technique for identifying infants at high risk of subsequent brain damage who might benefit from interventionist treatment after asphyxia.

(Arch Dis Child 1995; 72: F34-F38)

Keywords: birth asphyxia, EEG monitoring, outcome, full term infants.

Therapeutic intervention to improve cerebral outcome after birth asphyxia may soon become possible. ${ }^{1-3}$ But it probably needs to be instituted as soon as possible after the hypoxicischaemic insult. The treatment may have side effects so only infants at high risk of subsequent brain damage should be considered. ${ }^{34}$ Techniques for accurate risk estimation early in the postasphyxial course are therefore urgently required.

Apgar scores and measurements of fetal blood gases are widely used, but both methods are considered poor predictors of outcome after birth asphyxia. ${ }^{5-9}$ Clinical assess- ment of the degree of hypoxic-ischaemic encephalopathy (HIE) is a better predictor of outcome, but may not become obvious until 12-36 hours after birth. ${ }^{51011}$ Cranial tomography, somatosensory evoked potentials, and magnetic resonance spectroscopy (MRS) are useful for prognosis, but not until 24 hours or more after birth. ${ }^{12-14}$ Abnormal cerebral blood flow velocity measured with the Doppler technique has a high positive predictive value when performed after one to two days of life but has not been evaluated during the first postnatal hours. ${ }^{15}$

The cerebroelectrical background activity, recorded with conventional electroencephalography (EEG), is a sensitive prognostic indicator after asphyxia when recorded during the first week of life. ${ }^{16} 17$ However, practical problems will preclude standard EEG recordings within the first hours after birth, especially if this occurs during the night or a weekend. The amplitude integrated EEG (aEEG) technique has been developed to monitor cerebral electrical background activity in the intensive care unit. ${ }^{18}$ It has been used for several years for continuous longterm monitoring in neonatal intensive care. ${ }^{19}$ The aEEG is a trend recording of cerebral electrical activity. It can be applied and interpreted on-line and at the bedside, around the clock, by neonatal staff after some training. Earlier studies have shown that the background activity, recorded with the aEEG, has a good concordance with tape recorded EEG and standard EEG. ${ }^{20} 21$ Prognostic evaluation can be elicited from the background patterns in the aEEG in asphyxiated infants. ${ }^{20}$ The present study aimed to determine if the aEEG technique was also suitable for very early prognosis after birth asphyxia.

\section{Methods}

Eighty two full term (gestational age 37-42 weeks) infants were treated for intrapartum asphyxia in our neonatal intensive care unit (NICU) between 1 January 1987 to 31 December 1991. The infants had at least one sign of intrapartum fetal distress (abnormal cardiotocography, meconium stained amniotic fluid, or low cord $\mathrm{pH}$ ), together with a five minute Apgar score of $<7$. They all needed resuscitation with positive pressure ventilation. Four infants had an Apgar score of $>6$ at five minutes but were included because they all needed vigorous resuscitation at five minutes. Forty seven of the infants (30 inborn, 17 outborn) had aEEG monitoring started before six 
Table 1 Complication of data on 47 full term infants with birth asphyxia

\begin{tabular}{|c|c|c|c|}
\hline & $N=$ & Mean $(S D)$ & Range \\
\hline $\begin{array}{l}\text { Gestational age (weeks) } \\
\text { Birth weight }(\mathrm{g})\end{array}$ & & $\begin{array}{r}40 \cdot 0(1 \cdot 4) \\
3534(560)\end{array}$ & $\begin{array}{c}37-42 \\
2420-4500\end{array}$ \\
\hline Umbilical cord $\mathrm{pH}$ & 17 & $6.92(0.21)$ & $6 \cdot 66-7 \cdot 24$ \\
\hline Umbilical catheter $\mathrm{pH}^{\star}$ & 15 & $7 \cdot 00(0 \cdot 17)$ & $6 \cdot 70-7 \cdot 24$ \\
\hline \multicolumn{4}{|l|}{ Apgar score } \\
\hline 1 minute & & $2 \cdot 1(1 \cdot 5)$ & $1-7$ \\
\hline 5 minutes & & $4 \cdot 1(1 \cdot 8)$ & $1-8$ \\
\hline Resuscitation with positive pressure ventilation & 47 & & \\
\hline Mechanical ventilation & 33 & & \\
\hline HIE stage II-III $\dagger$ & $24(9 \ddagger)$ & & \\
\hline Spontaneous breathing & 14 & & \\
\hline HIE stage $0 t$ & 9 & & \\
\hline HIE stage I $\dagger$ & 5 & & \\
\hline aEEG at $0-6$ hours & 47 & & \\
\hline Standard EEG within 24 hours & 23 & & \\
\hline
\end{tabular}

${ }^{\star} \mathrm{pH}$ from umbilical catheter (arterial or venous) during resuscitation, before buffer, in infants with no $\mathrm{pH}$ measured from umbilical cord.

tHIE staging according to Sarnat and Sarnat ${ }^{10}$.

¥Nine infants could not be staged due to sedation with phenobarbital and diazepam.

hours of life and were included in the present study. The most common reason for an aEEG not being applied early in the 35 infants who were not included in the study was referral from other hospitals after the first six postnatal hours.

A

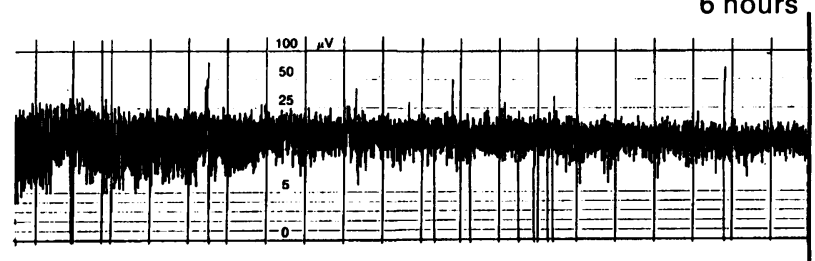

B

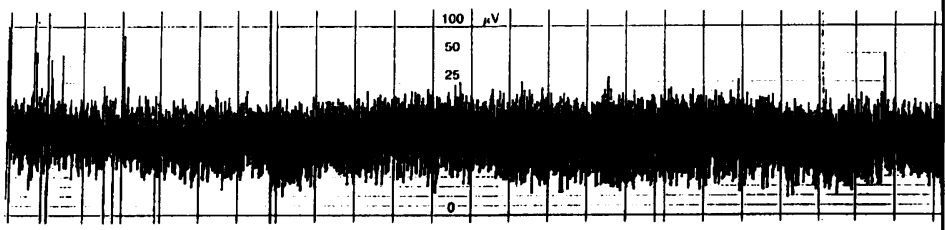

C

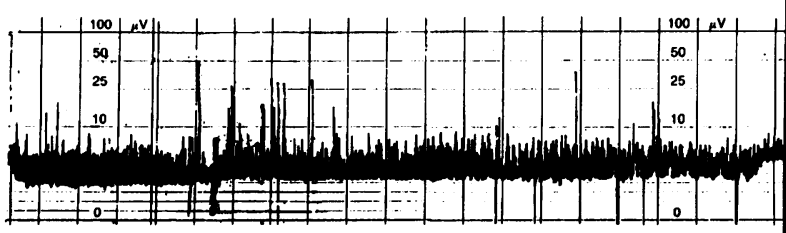

D

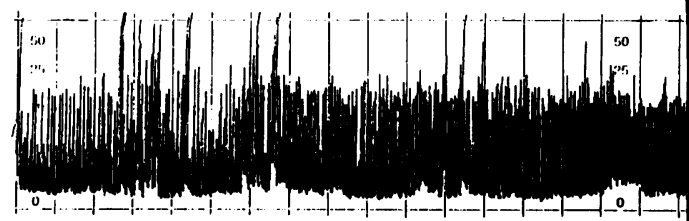

$E$

30 minutes

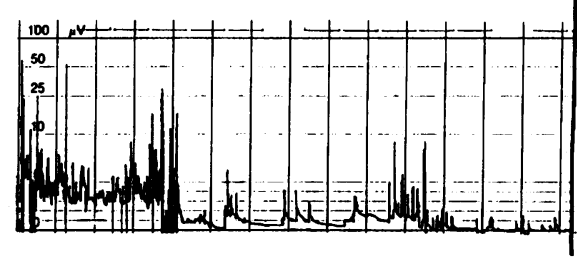

Amplitude integrated EEG from cerebral function monitor, showing four types of background patterns recorded from full term asphyxiated new born infants during the first six postnatal hours. Note semilogarithmic write-out of amplitude, and compressed time-scale. (A) Continuous normal voltage: the variation in band width is suggestive of sleep-wake cycles. (B) Continuous normal voltage in infant who received phenobarbital before the recording. No clear sleep-wake cycles. (C) Continuous extremely low voltage pattern, with suspected seizure pattern emerging during the last 40 minutes of the recording. (D) Suppression-burst pattern. (E) Flat tracing, mainly isoelectric.
Data on the babies studied are presented in table 1 . Thirty three of the 47 infants needed respiratory support with mechanical ventilation during the first six hours after birth. Twenty four of the 33 mechanically ventilated infants had clinically obvious hypoxic-ischaemic encephalopathy of various degrees, ${ }^{10}$ while the presence of HIE at $0-6$ hours was not possible to evaluate in nine of these infants because of sedation (mainly phenobarbital and diazepam). Fourteen infants were breathing spontaneously; five had clinical signs corresponding to mild HIE. Standard EEGs were recorded in 44 of the 47 infants on one to seven (mean 2.1) occasions during the neonatal period. In 23 infants the first EEG was recorded within 24 hours of birth.

After admission to the NICU, an amplitude integrated continuous EEG recording (aEEG) (Cerebral Function Monitor, CFM 4640, Lectromed, Devices Ltd) was applied by the attending nurse or neonatologist. The aEEG records a single channel EEG from two biparietal electrodes. The filtered signal, containing the main EEG frequencies, is rectified, smoothed, and amplitude integrated before it is written out, cot-side, on slow speed paper (6 or $30 \mathrm{~cm} /$ hour). The technique, which gives a trend recording of cerebroelectrical background activity, has been fully described elsewhere. ${ }^{18}$

The aEEG tracings were analysed according to: (i) main type of background activity, (a) continuous normal voltage, ${ }^{22}$ (b) burstsuppression, (c) continuous extremely low voltage (main activity below $5 \mu \mathrm{V}$ ), and (d) flat (mainly isoelectric); and (ii) presence of seizure patterns during the first six postnatal hours. The continuous and discontinuous patterns are illustrated in the figure. For the recordings, a paper speed of $6 \mathrm{~cm} /$ hour was used. To exclude bias in the interpretation only the part recorded during the first three and six hours of life was visible and evaluated by one of the authors (IR), who did not know the patient's identity and outcome. The working hypothesis was that, in the aEEG and in the standard EEG, a continuous background pattern of normal voltage predicted a good outcome. The other three types of tracings (suppression-burst, continuous extremely low voltage, and flat) were regarded as abnormal and predictive of poor outcome (handicap or death).

The influence of a loading dose of phenobarbital on the cerebroelectrical background activity was evaluated in 22 infants who were healthy at subsequent follow up. The minimum and maximum voltages between 5 and 6 hours of age in the aEEG tracings were compared between infants receiving a bolus of phenobarbital $(9-15 \mathrm{mg} / \mathrm{kg}$ ) and infants without any sedative medication.

The main type of background activity was compared in the early aEEG recording and the first standardised EEG (recorded within four days of life in 44 infants).

Outcome was evaluated from patient records in all infants. The handicapped infants were followed up by paediatric neurologists. 
Table 2 Predictive ability of early aEEG on outcome after birth asphyxia

\begin{tabular}{|c|c|c|c|c|}
\hline & $\begin{array}{l}\text { Sensitivity } \\
(\%)\end{array}$ & $\begin{array}{l}\text { Specificity } \\
(\%)\end{array}$ & $\begin{array}{l}\text { Positive predictive } \\
\text { value (\%) }\end{array}$ & $\begin{array}{l}\text { Efficiency } \\
\text { (\%) }\end{array}$ \\
\hline $\begin{array}{l}\text { aEEG (<6 hours) ( } n=47) \\
\text { Normal pattern (good outcome) } \\
\text { Abnormal pattern (poor outcome) } \\
\text { Normal + abnormal }\end{array}$ & $\begin{array}{l}89 \cdot 0 \\
94 \cdot 7\end{array}$ & $\begin{array}{l}94 \cdot 7 \\
89 \cdot 3\end{array}$ & $\begin{array}{l}96 \cdot 2 \\
85 \cdot 7\end{array}$ & 91.5 \\
\hline $\begin{array}{l}\text { Standard EEG (1-4 days) }(n=44) \\
\text { Normal pattern (good outcome) } \\
\text { Abnormal pattern (poor outcome) } \\
\text { Normal + abnormal }\end{array}$ & $\begin{array}{l}84 \cdot 6 \\
84 \cdot 2\end{array}$ & $\begin{array}{l}94 \cdot 1 \\
88 \cdot 0\end{array}$ & $\begin{array}{l}95 \cdot 6 \\
84 \cdot 2\end{array}$ & $90 \cdot 5$ \\
\hline
\end{tabular}

Normal pattern $=$ continuous with normal voltage.

Abnormal pattern=continuous with extremely low voltage, suppression-burst, and flat.

Most infants with a good short term outcome were followed up in the neonatal outpatient clinic for 12-18 months. some infants without encephalopathy, or Sarnat I HIE, were followed up for only a few months. To the infants with a good short term outcome, a questionnaire was sent to the parents asking whether the child had continued to develop normally and was doing well, and whether the results of routine health controls (routine for all Swedish children, and includes hearing and psychomotor evaluation at 18 months and 4 years of age as well as visual testing at 4 years) were normal. Outcome was divided into three groups: (1) healthy (including minor visual or hearing deficits); (2) neurological sequelae (cerebral palsy of any degree, psychomotor retardation requiring special training); (3) death (neonatal or later as a result of the sequelae of asphyxia).

\section{STATISTICAL METHODS}

For group comparisons, the Mann-Whitney $\mathrm{U}$ test (two-tailed) was used, and a $\mathrm{p}$ value of $<0.05$ was regarded as significant. For estimation of predictive ability the sensitivity, specificity, and positive predictive values of the different items were calculated. ${ }^{15} \mathrm{We}$ also estimated the 'efficiency' $(\%)$ as the total number of correctly predicted individuals in relation to the total number of observations made (true positive + true negative/all $\times 100$ ).

\section{Results}

AEEG BACKGROUND PATTERN AND LATER OUTCOME

Twenty six infants had a continuous background pattern of normal voltage during the first six hours after birth. Twenty five of these 26 infants had a good outcome, with normal development at a median age of 2 years and 9 months (range 12 months to 6 years). One infant needed special training for delayed psychomotor development at the age of 4 years.

Twenty one infants had abnormal aEEG patterns. Nine of the infants died, and nine survived with handicap; three infants survived and were healthy at follow up. Five infants had flat tracings; four of these infants died, while one survived with severe handicap. Two infants with continuous but extremely low voltage patterns survived with severe handicap. Nine of the 14 infants with suppression-burst patterns survived; six of these infants were handicapped while three survived without neurological sequelae. Table 2 shows the sensitivity, specificity, and positive predictive values for the different aEEG patterns in predicting subsequent outcome.

The ability of the aEEG to predict outcome earlier in the postasphyxial course - within the first three hours of life - was further evaluated in 19 infants who had been monitored for more than one hour during the first three postnatal hours. The prognostic efficiency of the aEEG recordings during the first three hours was lower $(14 / 19$ or $74 \%)$ than during the first six postnatal hours $(91 \cdot 5 \%)$. An initially continuous pattern in one infant changed into a very low voltage pattern at six hours. The aEEG tracing of three infants had improved between three and six hours, from suppression-burst to continuous patterns. One infant had a suppression-burst pattern at both three and six hours but survived without handicap.

\section{SEIZURES DURING THE FIRST SIX POSTNATAI} HOURS

Repetitive seizures within the first six hours, diagnosed in seven infants, were significantly correlated with poor outcome $(p=0.0004)$. Six of the infants had repetitive clinical seizures (mainly multifocal myoclonic). In these six infants the background activity of the aEEG was severely depressed and showed no corresponding electrographical seizure patterns. Five of the infants died and one survived with severe neurological handicap. One infant had repetitive subclinical seizures on the aEEG and survived with severe sequelae. Fourteen infants had suspected single clinical or subclinical seizures during the first six hours, with a poor outcome (sequelae or death) in seven; the other seven infants had a favourable outcome $(p=0.90)$.

\section{COMPARISON OF BACKGROUND ACTIVITY IN}

EARLY AEEG $v$ FIRST STANDARD EEG

Forty four of the 47 infants had standard EEGs recorded during the first four days of life (table 3). In 34 infants the type of background activity was similar in the aEEG and in the standard EEG. In four infants the initial suppression-burst patterns in the aEEG had deteriorated into isoelectric tracings in the EEG. In another four infants the standard EEG was normal (after two to four days) when the initial aEEG showed suppression-burst. In two infants, who were later healthy, the initial aEEG was continuous with normal voltage but the subsequent recorded standard EEG showed an intermittent, but not entirely

Table 3 Comparison between aEEG monitoring 0-6 hours and standard EEG on days 1-4 after birth in 44 infants

\begin{tabular}{llr}
\hline aEEG & Standard EEG 1-4 days & $N=$ \\
\hline Pattern and findings $\rightarrow$ & Similar in both & 34 \\
Suppression-burst $\rightarrow$ & Isoelectric & 4 \\
Suppression-burst $\rightarrow$ & Normal & 4 \\
Normal $\rightarrow$ & Discontinuous & $2^{\star}$ \\
\hline
\end{tabular}

$\star$ Both infants had normal outcome at 2 years of age. 
continuous, pattern on the first day of life. The efficiency of the standard EEG (performed during the first four days) to predict outcome correctly was $86.4 \%(38 / 44)$. In the same 44 infants the aEEG monitoring correctly predicted subsequent outcome in $90.9 \%(40 / 44)$.

\section{INFLUENCE OF PHENOBARBITAL ON THE AEEG AMPLITUDE}

In 22 infants, healthy at follow up, the amplitude of the background activity was compared between infants without neurosedative medication $(n=12)$ and infants receiving a loading dose of phenobarbital of 9-15 mg/kg $(n=10)$. In none of these infants was the aEEG applied before the phenobarbital was given. The background pattern was discontinuous (suppression-burst) in three of the infants who received phenobarbital, while the other infants had continuous background activity. The two groups of babies were the same for one, five, and 10 minute Apgar scores, cord $\mathrm{pH}$, first umbilical catheter $\mathrm{pH}$ and birth weight. The infants treated with phenobarbital had a significantly lower minimum $(5.5 v 10.4 \mu \mathrm{V}$; $\mathrm{p}=0.008)$ but unchanged maximum $(18.3 v$ $26.6 \mu \mathrm{V} ; \mathrm{p}=0.08$ ) amplitude compared with the infants without medication.

\section{Discussion}

These findings indicate that outcome can be predicted accurately after birth asphyxia in full term newborn infants during the first six postnatal hours with a continuous single channel aEEG. Postasphyxial intervention has included the use of potent medication. ${ }^{34}$ The benefits of such medication must be balanced against the risk of adverse effects. The decision as to which infants should be candidates for postasphyxial treatment should probably be made early after the primary insult, before the secondary cascade of negative intracellular and extracellular processes begin or are fully developed. ${ }^{123}$ In this respect the aEEG monitoring technique seems to be suitable for early identification of the infants at high risk for postasphyctic brain damage. The background pattern in the aEEG recordings predicted gross outcome correctly in $91.5 \%$ of the infants. The positive predictive value of the background activity in the aEEG agrees with earlier standard EEG studies recorded within the first week of life. ${ }^{101624-26}$ To our knowledge there have been no previous EEG studies performed after birth asphyxia at this very early postnatal age.

A continuous normal voltage pattern at 6 hours of postnatal age is almost always a good prognostic indicator: 25 of 26 infants with this background pattern survived without handicap in the present study. The only infant with normal background pattern but adverse outcome developed psychomotor retardation, for which he required special training, but no cerebral palsy. This infant showed no clinical signs of hypoxic-ischaemic encephalopathy in the NICU, and did not receive mechanical ventilation or neurosedative medication.
Consequently his developmental retardation could well have been attributable to causes other than intrapartum birth asphyxia.

Eighteen of the $21(86 \%)$ infants with abnormal background activity at six postnatal hours had a poor outcome; nine of the infants died and nine survived with handicap. However, three infants with suppression-burst patterns survived without handicap. The three infants were all treated with phenobarbital before the aEEG recordings. The phenobarbital might have contributed to the depressed background activity in these infants. Consequently physicians need to be aware that the predictive value of a suppression-burst pattern is lower $(78.6 \%)$ than the predictive value for the other patterns. Still, most infants with suppression-burst within the first six hours will sustain severe neurological sequelae or will die. When all infants with good outcome were compared, the infants who had received phenobarbital before the aEEG recordings had a significantly lower minimum voltage. The phenobarbital might also change a normal continuous background pattern into a suppression-burst pattern. Still, it is our clinical impression that the cerebroelectrical background activity deteriorates more after neurosedative medication in a baby with a damaged brain than in a baby with an undamaged brain. Consequently, when evaluating cerebroelectrical background activity, the influence of neurosedative medications must always be considered.

In the present study early repetitive seizures (clinical or subclinical) were significantly correlated with poor outcome, while $50 \%$ of infants with single seizures had a good prognosis. However, only seven infants out of the total 19 with adverse outcome (handicap or death) had repetitive seizures. Repetitive seizures must therefore be regarded as highly selective for a group of very severely damaged infants, and the absence of repetitive seizures is not predictive of a good outcome.

The postnatal evaluation might need to be made earlier than at 6 hours of age. We evaluated the prognostic ability of the aEEG during the first three hours after birth, but the efficiency was lower (74\%) at this time. The lower accuracy could partly have been due to the lower number of infants recorded. However, in four of the 19 infants the type of background activity actually changed from a normal to an abnormal or vice versa, between three and six hours. This finding warrants further investigation in a larger series of infants recorded at an earlier postnatal age.

The predictive value of EEGs recorded after two to four days is probably lower than if the EEG is recorded within the first day. Still, the prognostic ability of the standard EEG, performed between 2 and 4 days of life, was about equal when compared with the six hour aEEG recordings. Later - after some days - it is usually clinically obvious which infants have developed severe HIE, but it is probably too late for intervention. ${ }^{1}$

During the past few years, the clinical use of continuous cerebroelectrical monitoring in sick 
neonates has increased. 192728 The EEG monitor should therefore be reliable, easy to apply and interpret (be a normal part of the monitoring of a sick baby), and give an overview of longterm trends in cerebroelectrical background activity. Different types of monitors are available, based on amplitudeintegration or frequency analysis. For a sick or a preterm newborn infant with a high risk of a discontinuous background pattern, a system based on the amplitude-integration seems to be the most feasible technique. ${ }^{29}$ The aEEG gives a longterm, bedside, and on-line trend recording of cerebroelectrical background activity but does not give information on localisation. ${ }^{18}$ Changes in the background activity, such as sleep-wake cycles, and repeated seizure activity are usually easy to identify after some training. However, in most infants we performed both aEEG and standard EEG. The two techniques are used together because they give different types of information.

In conclusion, the present data indicate that an early postnatal evaluation, including antenatal data and clinical signs of HIE, together with aEEG monitoring during the first six hours of life, can give a rapid and reliable determination of the severity of HIE after birth asphyxia. The data could help indicate which infants might require more specific therapeutic programmes in the near future.

This study was supported by grants from the Swedish Medical Research Council (grant No 4732 and 084 ), Lund University Research Council (grant No 4732 and 084 ), Lund University
Medical Faculty, Kock Foundation, and the Josef and Linnea Carlsson Foundation.

1 Levene MI. Management of the asphyxiated full term infant. Arch Dis Child 1993; 68: 612-6.

2 Thiringer K. Hrbek A, Karlsson K, Rosén KG, Kjellmer I. Postasphyxial cerebral survival in newborn sheep after treatment with oxygen free radical scavengers and a calcium antagonist. Pediatr Res 1987; 22: 62-6.

3 Vannucci RC. Current and potentially new management strategies for perinatal hypoxic-ischemic encephalopathy. Pediatrics 1990; 85: 961-8.

4 Levene MI, Gibson NA, Fenton AC, Papathoma E, Barnett D. The use of a calcium-channel blocker, nicardipine, for severely asphyxiated newborn infants. Dev Med Child Neurol 1990; 30: 567-74.

5 Nelson KB, Ellenberg JH. Apgar scores as predictors of chronic neurologic disability. Pediatrics 1981; 68: 36-44.

6 Levene MI, Sands C, Grindulis H, Moore JR. Comparison of two methods of predicting outcome in perinatal of two methods of predicting

7 Freeman JM, Nelson KB. Intrapartum asphyxia and cerebral palsy. Pediatrics 1988; 82: 240-9.

8 Finer NN, Robertson CM, Peters KL, Coward JH. Factors affecting outcome in hypoxic-ischemic encephalopathy in term infants. Arch Dis Child 1983; 137: 21-5.
9 Ruth VJ, Raivio KO. Perinatal brain damage: Predictive value of metabolic acidosis and Apgar score: $B M F$ 1988; 297: 24-7.

10 Sarnat HB, Sarnat MS. Neonatal encephalopathy following fetal distress. A clinical and electroencephalographic study. Arch Neurol 1976; 33: 696-705.

11 Robertson C, Finer N. Term infants with hypoxic-ischemic encephalopathy: Outcome at 3.5 years. Dev Med Child Neurol 1985; 27: 473-84.

12 de Vries LS, Pierrat V, Eken P, Minami T, Daniels H, Casaer P. Prognostic value of early somatosensory evoked potentials for adverse outcome in full-term infants with potentials for adverse outcome in full-term
birth asphyxia. Brain Dev 1991; 13: 320-5.

13 Azzopardi D, Wyatt JS, Cady EB, Delpy BT, Baudin J, Stewart AL, et al. Prognosis of newborn infants with hypoxic-ischemic brain injury assessed by phosphorus magnetic resonance spectroscopy. Pediatr Res 1989; 25: 445-51.

14 Fitzhardinge PM, Flodmark O, Fitz CR, Ashby S. The prognostic value of computed tomography as an adjunct to assessment of the term infants with postasphyxial encephalopathy. F Pediatr 1981; 99: 777-81.

15 Levene MI, Fenton AC, Evans DH, Archer LNJ, Shortland DB, Gibson NA. Severe birth asphyxia and abnormal cerebral blood-flow velocity. Dev Med Child Neurol 1989; 31: 427-34.

16 Holmes G, Rowe J, Hafford J, Schmidt R, Testa M, Zimmerman A. Prognostic value of the electroencephalogram in neonatal asphyxia. Electroencephalogr Clin Neurophysiol 1982; 53: 60-72.

17 Wayenberg J-L, Vermeylen D, Bormans J, Denis R. Early prediction of perinatal neurological evolution in term asphyxiated newborns. F Perinat Med 1992; 20 (suppl 1): 242.

18 Prior PF, Maynard DE. Monitoring cerebral function. Longterm recordings of cerebral electrical activity and evoked potentials. Amsterdam: Elsevier, 1986.

19 Hellström-Westas L, Rosén I, Svenningsen NW. Silent seizures in sick infants in early life. Acta Paediatr Scand 1985; 74: 741-8.

20 Bjerre I, Hellström-Westas L, Rosén I, Svenningsen NW. Monitoring of cerebral function after severe asphyxia in infancy. Arch Dis Child 1983; 58: 997-1002.

21 Hellstöm-Westas L. Comparison between tape-recorded and amplitude-integrated EEG in sick neonates. Acta Paediatr 1993; 81: 812-8.

22 Thornberg E, Thiringer $\mathrm{K}$. Normal patterns of cerebral function monitor traces in term and preterm neonates. Acta Paediatr Scand 1990; 79: 20-5.

23 Kjellmer I. Prenatal and intrapartum asphyxia. In: Levene MI, Bennett MJ, Punt J, eds. Fetal and neonatal neurology and neurosurgery. London: Churchill Livingstone, 1988: and neur 69 .

24 Pezzani C, Radvanyi-Bouvet MF, Relier JP, Monod N. Neonatal encephalography during the first 24 hours of life in fullterm newborn infants. Neuropediatrics 1986; 17: 11-8.

25 Monod N, Pajot N, Guidasci S. The neonatal EEG: statistical studies and prognostic value in fullterm and preterm babies. Electroencephalogr Clin Neurophysiol 1972; 32: 529-44.

26 Watanabe K, Miyazaki S, Hara K, Hakamada S. Behavioral state cycles, background EEGs and prognosis of newborns with perinatal hypoxia. Electroencephalogr Clin Neurophysiol 1980; 49: 618-25.

27 Eyre JA, Oozeer R, Wilkinson AR. Diagnosis of neonatal seizures by continuous recording and rapid analysis of the
electroencephalogram. Arch Dis Child 1983; 58: 785-9.

28 Connell I, Oozeer R, de Vries LS, Regan R, Dubowitz LMS, Dubowitz V. Continuous EEG monitoring of neonatal seizures: diagnostic and prognostic considerations. Arch Dis Child 1989; 64: 452-8.

29 Murdoch-Eaton D, Toet M, Livingston J, Smith I, Levene $M$. Monitoring cerebral function in the neonatal intensive care. Proceedings of the Neonatal Society. Birmingham, 1993: 5. 